\title{
Application of Functional Link Artificial Neural Network for Prediction of Machinery Noise in Opencast Mines
}

\author{
Santosh Kumar Nanda ${ }^{1}$ and Debi Prasad Tripathy ${ }^{2}$ \\ ${ }^{1}$ Department of Computer Science and Engineering, Eastern Academy of Science and Technology, Bhubaneswar, Orissa 754001, India \\ ${ }^{2}$ Department of Mining Engineering, National Institute of Technology, Rourkela, Orissa 769008, India
}

Correspondence should be addressed to Santosh Kumar Nanda, santosh.rsmining@gmail.com

Received 16 December 2010; Accepted 22 April 2011

Academic Editor: E. E. Kerre

Copyright (C) 2011 S. K. Nanda and D. P. Tripathy. This is an open access article distributed under the Creative Commons Attribution License, which permits unrestricted use, distribution, and reproduction in any medium, provided the original work is properly cited.

\begin{abstract}
Functional link-based neural network models were applied to predict opencast mining machineries noise. The paper analyzes the prediction capabilities of functional link neural network based noise prediction models vis-à-vis existing statistical models. In order to find the actual noise status in opencast mines, some of the popular noise prediction models, for example, ISO-9613-2, CONCAWE, VDI, and ENM, have been applied in mining and allied industries to predict the machineries noise by considering various attenuation factors. Functional link artificial neural network (FLANN), polynomial perceptron network (PPN), and Legendre neural network (LeNN) were used to predict the machinery noise in opencast mines. The case study is based on data collected from an opencast coal mine of Orissa, India. From the present investigations, it could be concluded that the FLANN model give better noise prediction than the PPN and LeNN model.
\end{abstract}

\section{Introduction}

At the present time, owing to the improvements in technology through superior energy competence, higher labor output, continuous production methods, and operating flexibility, automation has also advanced rapidly in open and underground pits together with mineral processing plants. In parallel to this improvement, sources of noise and ambient noise at work place in the mining industry have increased significantly. In general, noise is generated from all most all the opencast mining operations from different fixed, mobile, and impulsive sources, thereby becoming an integral part of the mining environment. With increased mechanization, the problem of noise has got accentuated in opencast mines. Prolonged exposure of miners to the high levels of noise can cause noise-induced hearing loss besides several nonauditory health effects [1]. The impact of noise in opencast mines depends upon the sound power level of the noise generators, prevailing geomining conditions and the meteorological parameters of the mines [2-4]. The noise levels need to be studied as an integrated effect of the above parameters. In mining conditions the equipment conditions and environment continuously change as the mining activity progresses. Depending on their placement, the overall mining noise emanating from the mines varies in quality and level. Thus, for environmental noise prediction models, the noise level at any receiver point needs to be the resultant sound pressure level of all the noise sources.

The need for accurately predicting the level of sound emitted in opencast mines is well established. Some of the noise forecasting models used extensively in Europe are those of the German draft standard VDI-2714 outdoor sound propagation and environmental noise model (ENM) of Australia [5]. These models are generally used to predict noise in petrochemical complexes, and mines. The algorithm used in these models rely for a greater part on interpolation of experimental data which is a valid and useful technique, but their applications are limited to sites which are more or less similar to those for which the experimental data were assimilated.

A number of models were developed and extensively used for the assessment of sound pressure level and their attenuation around industrial complexes. Generally, in Indian mining industry, environmental noise model developed by 
TABLE 1: Simulation study of shovel noise.

\begin{tabular}{|c|c|c|c|c|c|c|c|c|}
\hline \multirow{2}{*}{$\begin{array}{l}\text { Distance from the } \\
\text { source (meters) }\end{array}$} & \multirow{2}{*}{$\begin{array}{l}\text { Measured field } \\
\text { data }(\mathrm{dBA})\end{array}$} & \multicolumn{4}{|c|}{ Prediction result (dBA) } & \multicolumn{2}{|c|}{ Average percentage error (dBA) } & \multirow[b]{2}{*}{ LeNN } \\
\hline & & VDI & PPN & FLANN & LeNN & PPN & FLANN & \\
\hline 1 & 102.3000 & 95.6919 & 74.1198 & 88.6731 & 81.4871 & & & \\
\hline 2 & 102.1000 & 95.4828 & 74.1198 & 94.3428 & 81.4871 & & & \\
\hline 3 & 98.6000 & 91.9738 & 74.1198 & 94.5178 & 81.4871 & & & \\
\hline 4 & 98.2000 & 91.5648 & 74.1198 & 89.4572 & 81.4871 & & & \\
\hline 5 & 97.5000 & 90.8559 & 74.1198 & 87.9020 & 81.4871 & & & \\
\hline 6 & 97.5000 & 90.8469 & 85.8609 & 96.5394 & 94.5712 & & & \\
\hline 7 & 96.7000 & 90.0380 & 85.8609 & 90.3449 & 94.5712 & & & \\
\hline 8 & 95.2000 & 88.5291 & 85.8609 & 93.3190 & 94.5712 & & & \\
\hline 9 & 93.3000 & 86.6202 & 85.8609 & 94.0383 & 94.5712 & & & \\
\hline 10 & 92.4000 & 85.7113 & 85.8609 & 94.0383 & 94.5712 & & & \\
\hline 11 & 91.5000 & 84.8025 & 85.8609 & 87.6493 & 94.5712 & & & \\
\hline 12 & 91.5000 & 84.7937 & 85.8609 & 88.3686 & 94.5712 & & & \\
\hline 13 & 91.3000 & 84.5848 & 85.8609 & 85.2195 & 94.5712 & & & \\
\hline 14 & 90.4000 & 83.6760 & 85.8609 & 91.4140 & 94.5712 & & & \\
\hline 15 & 88.8000 & 82.0672 & 76.5756 & 85.3685 & 85.3813 & 7.03 & 5.68 & 8.42 \\
\hline 16 & 88.4000 & 81.6585 & 76.5756 & 85.3685 & 85.3813 & & & \\
\hline 17 & 87.9000 & 81.1497 & 76.5756 & 88.5889 & 85.3813 & & & \\
\hline 18 & 87.1000 & 80.3410 & 76.5756 & 88.4139 & 85.3813 & & & \\
\hline 19 & 86.7000 & 79.9323 & 76.5756 & 87.6947 & 85.3813 & & & \\
\hline 20 & 86.3000 & 79.5236 & 76.5756 & 87.6947 & 85.3813 & & & \\
\hline 21 & 85.7000 & 78.9149 & 76.5756 & 85.1287 & 85.3813 & & & \\
\hline 22 & 85.2000 & 78.4063 & 74.3142 & 84.4095 & 85.3813 & & & \\
\hline 23 & 85.3000 & 78.4976 & 74.3142 & 84.4095 & 85.3813 & & & \\
\hline 24 & 85.5000 & 78.6890 & 73.2126 & 81.1891 & 85.3813 & & & \\
\hline 25 & 85.5000 & 78.6804 & 73.2126 & 80.1264 & 82.0894 & & & \\
\hline 26 & 85.3000 & 78.4718 & 75.3380 & 81.6816 & 82.0894 & & & \\
\hline 27 & 84.7000 & 77.8632 & 71.4890 & 81.4353 & 82.0894 & & & \\
\hline 28 & 84.2000 & 77.3547 & 72.2342 & 81.6103 & 82.7253 & & & \\
\hline 29 & 83.8000 & 76.9461 & 72.2342 & 82.3295 & 82.9287 & & & \\
\hline 30 & 82.7000 & 75.8376 & 72.2342 & 82.3295 & 82.9287 & & & \\
\hline
\end{tabular}

RTA group, Australia is mostly used to predict noise $[4,6]$. ENM was used to predict sound pressure level in mining complexes at Moonidih Project in Jharia Coalfield, Dhanbad, India [6]. The applied model output was represented as noise contours. The application of different noise prediction models was studied for various mines and petrochemical complexes and it was reported that VDI2714 model was the simplest and least complex model vis-à-vis other models [5]. VDI2714 and ISO (1996) noise prediction models were used in Assiut cement plant, Assiut cement quarry and ElGedida mine at El-Baharia oasis of Egypt to predict noise. From the study, it was concluded that the prediction models could be used to identify the safe zones with respect to the noise level in mining and industrial plants. It was also inferred that the VDI2714 model is the simplest model for prediction of noise in mining complexes and workplace [7]. Air attenuation model was developed for noise prediction in limestone quarry and mines of Ireland. The model was used to predict attenuation in air due to absorption [8].

All the noise prediction models treat noise as a function of distance, sound power level, different form of attenuations such as geometrical absorptions, barrier effects, ground topography. Generally, these parameters are measured in the mines and best fitting models are applied to predict noise. Mathematical models are generally complex and cannot be implemented in real time systems. Additionally, they fail to predict the future parameters from current and past measurements. It has been seen that noise prediction is a nonstationary process and soft-computing techniques like fuzzy system, adaptive neural network-based fuzzy inference system (ANFIS), neural network, and so forth, have been tested for nonstationary time-series prediction nearly for two decades. There is a scope of using different soft computing techniques: fuzzy logic, artificial neural networks, radial basis function (RBF) and so forth, for noise prediction in mines. In comparison to other soft computing techniques, functional link artificial neural network (FLANN) and Legendre Neural Network (LeNN) has less computational cost and easily implemented in hardware applications. This is the motivation on which the present research work is based.

In this paper, an attempt has been made to develop three types of functional link artificial neural network models 


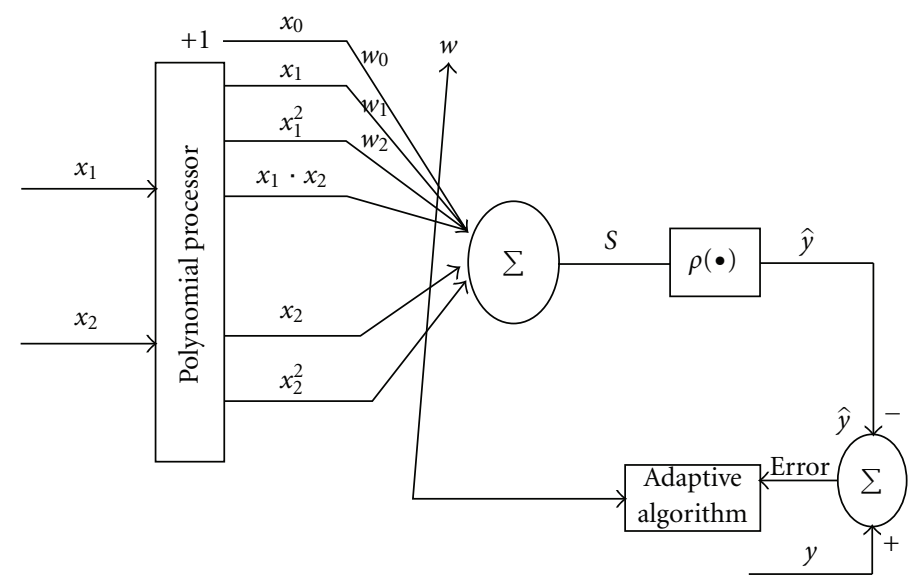

(a) PPN structure

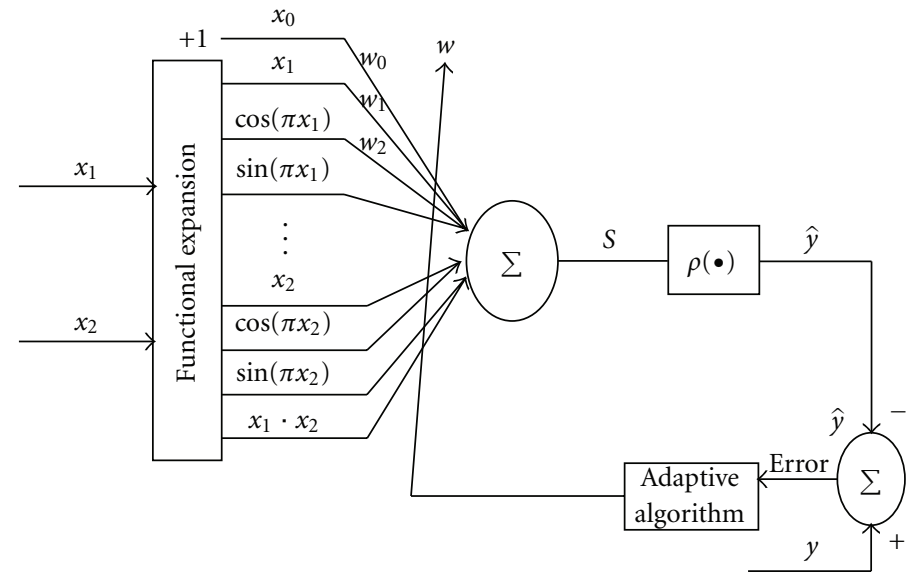

(b) FLANN structure

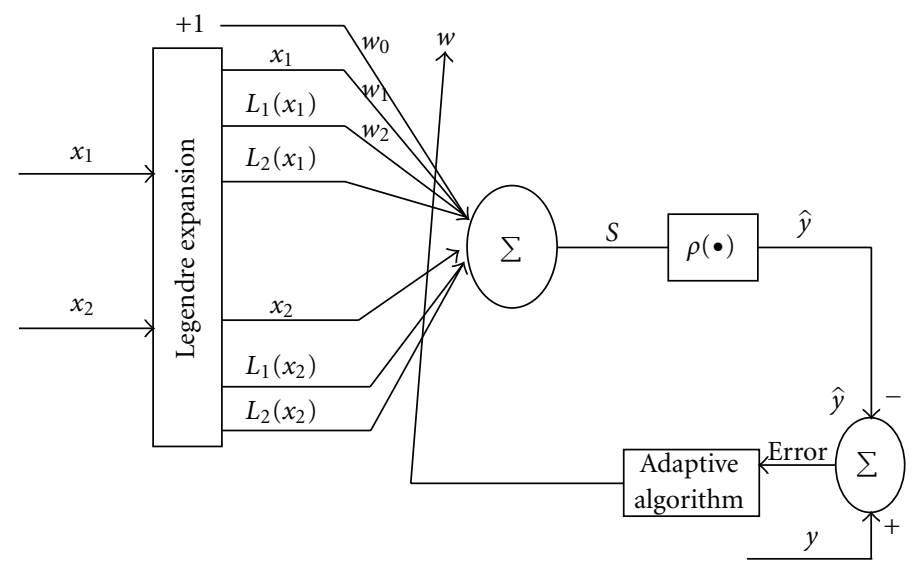

(c) Legendre neural network structure

FIGURE 1: System architecture of function link based artificial neural networks.

(FLANN, PPN, and LeNN) for noise prediction of machineries used in Balaram opencast coal mine of Talcher, Orissa, India. The data assembled through surveys, measurement or knowledge to predict sound pressure level in mines is often imprecise or speculative. Since neural network-based systems are good predictive tools for imprecise and uncertainty information; therefore, the proposed approach would be the most appropriate technique for modeling the prediction of sound pressure level in opencast coal mines.

\section{VDI-2714 Noise Prediction Model}

In 1976, the VDI draft code 2714 "outdoor sound propagation" was issued by the VDI committee on noise reduction 
TABle 2: Simulation study of dumper noise.

\begin{tabular}{|c|c|c|c|c|c|c|c|c|}
\hline \multirow{2}{*}{$\begin{array}{l}\text { Distance from the } \\
\text { source (meters) }\end{array}$} & \multirow{2}{*}{$\begin{array}{l}\text { Measured field } \\
\text { data }(\mathrm{dBA})\end{array}$} & \multicolumn{4}{|c|}{ Prediction result (dBA) } & \multicolumn{3}{|c|}{ Average percentage error (dBA) } \\
\hline & & VDI & PPN & FLANN & LeNN & PPN & FLANN & LeNN \\
\hline 1 & 102.4000 & 95.7919 & 74.1198 & 88.6731 & 81.4871 & & & \\
\hline 2 & 101.3000 & 94.6828 & 74.1198 & 93.0404 & 81.4871 & & & \\
\hline 3 & 98.2000 & 91.5738 & 74.1198 & 94.5178 & 81.4871 & & & \\
\hline 4 & 97.7000 & 91.0648 & 74.1198 & 89.4572 & 81.4871 & & & \\
\hline 5 & 97.2000 & 90.5559 & 74.1198 & 87.9020 & 81.4871 & & & \\
\hline 6 & 96.8000 & 90.1469 & 85.8609 & 96.5394 & 94.5712 & & & \\
\hline 7 & 94.2000 & 87.5380 & 74.1198 & 82.7701 & 81.4871 & & & \\
\hline 8 & 94.1000 & 87.4291 & 85.8609 & 93.3190 & 94.5712 & & & \\
\hline 9 & 93.6000 & 86.9202 & 85.8609 & 94.0383 & 94.5712 & & & \\
\hline 10 & 93.2000 & 86.5113 & 85.8609 & 94.0383 & 94.5712 & & & \\
\hline 11 & 93.2000 & 86.5025 & 85.8609 & 84.6752 & 94.5712 & & & \\
\hline 12 & 92.5000 & 85.7937 & 85.8609 & 85.3944 & 94.5712 & & & \\
\hline 13 & 92.2000 & 85.4848 & 85.8609 & 86.3534 & 94.5712 & & & \\
\hline 14 & 90.6000 & 83.8760 & 85.8609 & 88.4398 & 94.5712 & & & \\
\hline 15 & 89.7000 & 82.9672 & 76.5756 & 85.3685 & 85.3813 & 7.27 & 5.77 & 8.20 \\
\hline 16 & 88.3000 & 81.5585 & 76.5756 & 85.3685 & 85.3813 & & & \\
\hline 17 & 88.2000 & 81.4497 & 76.5756 & 88.5889 & 85.3813 & & & \\
\hline 18 & 87.6000 & 80.8410 & 76.5756 & 88.4139 & 85.3813 & & & \\
\hline 19 & 87.1000 & 80.3323 & 76.5756 & 87.6947 & 85.3813 & & & \\
\hline 20 & 86.8000 & 80.0236 & 76.5756 & 87.6947 & 85.3813 & & & \\
\hline 21 & 86.5000 & 79.7149 & 76.5756 & 85.1287 & 85.3813 & & & \\
\hline 22 & 86.2000 & 79.4063 & 74.3142 & 84.4095 & 85.3813 & & & \\
\hline 23 & 85.8000 & 78.9976 & 74.3142 & 84.4095 & 85.3813 & & & \\
\hline 24 & 85.6000 & 78.7890 & 73.2126 & 81.1891 & 85.3813 & & & \\
\hline 25 & 84.8000 & 77.9804 & 73.2126 & 83.1006 & 82.0894 & & & \\
\hline 26 & 84.2000 & 77.3718 & 75.3380 & 84.6557 & 82.0894 & & & \\
\hline 27 & 84.2000 & 77.3632 & 71.4890 & 81.4353 & 82.0894 & & & \\
\hline 28 & 83.7000 & 76.8547 & 72.0592 & 81.6103 & 82.7253 & & & \\
\hline 29 & 83.4000 & 76.5461 & 72.2342 & 82.3295 & 82.9287 & & & \\
\hline 30 & 82.8000 & 75.9376 & 72.2342 & 82.3295 & 82.9287 & & & \\
\hline
\end{tabular}

[5]. The sound pressure level at an environmental point is calculated from the following equation (1)

$$
\begin{array}{r}
L_{P} \mathrm{~dB}(\mathrm{~A})=\sum_{\text {all sources }}^{\log }\left[L_{W}+K_{1}-10 \log \left(4 \pi R^{2}\right)+3 \mathrm{~dB}\right. \\
\left.-K_{2}-K_{3}-K_{4}-K_{5}-K_{6}-K_{7}\right],
\end{array}
$$

$L_{w}$ : source power level re $10^{-12}$ watts, $K_{1}$ : source directivity index, $-10 \log \left(4 \pi R^{2}\right)$ : geometric spreading term including infinite hard plane coinciding with the source, $R$ : source to receiver distance, $K_{2}$ : atmospheric attenuation $=10 \log (1+$ $0.0015 R) \mathrm{dB}(\mathrm{A}), K_{3}$ : attenuation due to meteorological conditions $=\left[\left(12.5 / R^{2}\right)+0.2\right]^{-1} \mathrm{~dB}(\mathrm{~A}), K_{4}$ : ground effects = $10 \log [3+(R / 160)]-K_{2}-K_{3} \mathrm{~dB}(\mathrm{~A}), K_{5}$ : barrier value $(0-$ $10)=10 \log (3+20 d) \mathrm{dB}(\mathrm{A}), d$ : barrier path difference, $K_{6}$ : attenuation due to woodland areas, $K_{7}$ : attenuation due to built-up areas.

\section{Introduction to Functional Link-Based Artificial Neural Network}

Neural network (NN) represents an important paradigm for classifying patterns or approximating complex nonlinear process dynamics. These properties clearly indicate that NN exhibit some intelligent behavior, and are good candidate models for nonlinear processes, for which no perfect mathematical model is available. Neural networks have been a powerful tool for their applications for more than last two decades [9-13]. Multilayer perceptron (MLP), radial basis function (RBF), Support vector machine (SVM) and so forth, are the types of Neural Network Model, where these models have better prediction competence with high computational cost. Generally, these models have high computational cost due to the availability of hidden layer. To minimize the computational cost, structures like, polynomial perceptron network (PPN) [14], functional link artificial neural network (FLANN) [15-18], Legendre neural 
TABLE 3: Simulation study of grader noise.

\begin{tabular}{|c|c|c|c|c|c|c|c|c|}
\hline \multirow{2}{*}{$\begin{array}{l}\text { Distance from the } \\
\text { source (meters) }\end{array}$} & \multirow{2}{*}{$\begin{array}{l}\text { Measured field } \\
\text { data }(\mathrm{dBA})\end{array}$} & \multicolumn{4}{|c|}{ Prediction result (dBA) } & \multicolumn{3}{|c|}{ Average percentage error (dBA) } \\
\hline & & VDI & PPN & FLANN & LeNN & PPN & FLANN & LeNN \\
\hline 1 & 105.3000 & 98.6919 & 74.1198 & 89.9755 & 81.4871 & & & \\
\hline 2 & 103.4000 & 96.7828 & 74.1198 & 95.4768 & 81.4871 & & & \\
\hline 3 & 101.2000 & 94.5738 & 74.1198 & 92.6776 & 81.4871 & & & \\
\hline 4 & 98.7000 & 92.0648 & 74.1198 & 89.4572 & 81.4871 & & & \\
\hline 5 & 97.2000 & 90.5559 & 74.1198 & 87.9020 & 81.4871 & & & \\
\hline 6 & 95.5000 & 88.8469 & 74.1198 & 85.9905 & 81.4871 & & & \\
\hline 7 & 94.3000 & 87.6380 & 74.1198 & 82.7701 & 81.4871 & & & \\
\hline 8 & 94.1000 & 87.4291 & 85.8609 & 93.3190 & 94.5712 & & & \\
\hline 9 & 93.7000 & 87.0202 & 85.8609 & 94.0383 & 94.5712 & & & \\
\hline 10 & 93.2000 & 86.5113 & 85.8609 & 94.0383 & 94.5712 & & & \\
\hline 11 & 92.6000 & 85.9025 & 85.8609 & 87.6493 & 94.5712 & & & \\
\hline 12 & 91.8000 & 85.0937 & 85.8609 & 88.3686 & 94.5712 & & & \\
\hline 13 & 90.4000 & 83.6848 & 85.8609 & 88.1936 & 94.5712 & & & \\
\hline 14 & 88.6000 & 81.8760 & 85.8609 & 91.4140 & 94.5712 & & & \\
\hline 15 & 88.5000 & 81.7672 & 76.5756 & 85.3685 & 85.3813 & 9.56 & 6.15 & 9.76 \\
\hline 16 & 88.2000 & 81.4585 & 76.5756 & 85.3685 & 85.3813 & & & \\
\hline 17 & 87.9000 & 81.1497 & 76.5756 & 88.5889 & 85.3813 & & & \\
\hline 18 & 87.3000 & 80.5410 & 76.5756 & 88.4139 & 85.3813 & & & \\
\hline 19 & 86.5000 & 79.7323 & 76.5756 & 87.6947 & 85.3813 & & & \\
\hline 20 & 85.8000 & 79.0236 & 76.5756 & 87.6947 & 85.3813 & & & \\
\hline 21 & 85.4000 & 78.6149 & 76.5756 & 85.1287 & 85.3813 & & & \\
\hline 22 & 85.1000 & 78.3063 & 74.3142 & 84.4095 & 85.3813 & & & \\
\hline 23 & 84.6000 & 77.7976 & 74.3142 & 84.4095 & 85.3813 & & & \\
\hline 24 & 84.2000 & 77.3890 & 73.2126 & 81.1891 & 85.3813 & & & \\
\hline 25 & 83.8000 & 76.9804 & 73.2126 & 83.1006 & 82.0894 & & & \\
\hline 26 & 83.2000 & 76.3718 & 63.5968 & 74.1068 & 66.3679 & & & \\
\hline 27 & 82.9000 & 76.0632 & 71.4890 & 81.4353 & 82.0894 & & & \\
\hline 28 & 82.5000 & 75.6547 & 60.3181 & 71.0614 & 67.0982 & & & \\
\hline 29 & 82.1000 & 75.2461 & 60.3181 & 71.7806 & 67.3327 & & & \\
\hline 30 & 81.8000 & 74.9376 & 60.3181 & 71.7806 & 67.3327 & & & \\
\hline
\end{tabular}

network (LeNN) $[19,20]$ were proposed. In this paper, three types of functional based artificial neural networks have been applied to predict mining machinery noise. These include polynomial perceptron network (PPN), functional link artificial neural network (FLANN), and Legendre neural network (LeNN).

In general, the functional link-based neural network models were single-layer ANN structure possessing higher rate of convergence and lesser computational load than those of an MLP structure. The behavior and mapping ability of a PPN and its application to channel equalization is reported by Xiang et al. (1994) [14]. The mathematical expression and computational calculation is evaluated as per MLP. Figure 1(a) represents the structure of PPN. Patra originally proposed functional link artificial neural network (FLANN), and it is a novel single-layer ANN structure capable of forming arbitrarily complex decision regions by generating nonlinear decision boundaries [15-18]. In
FLANN, the hidden layers are removed. Further, the FLANN structure offers less computational complexity and higher convergence speed than those of an MLP because of its single-layer structure. The FLANN structure is depicted in Figure 1(b). Here, the functional expansion block makes use of a functional model comprising a subset of orthogonal sin and cos basis functions and the original pattern along with its outer products. For example, considering a two-dimensional input pattern $X=\left[x_{1} x_{2}\right]^{T}$. The enhanced pattern is obtained by using the trigonometric functions as $X^{*}=$ $\left[x_{1} \cos \left(\pi x_{1}\right) \sin \left(\pi x_{1}\right) \cdots x_{2} \cos \left(\pi x_{2}\right) \sin \left(\pi x_{2}\right) \cdots x_{1} x_{2}\right]^{T}$ which is then used by the network for the equalization purpose. The BP algorithm, which is used to train the network, becomes very simple because of absence of any hidden layer. Justification for the use of the trigonometric functions in the FLANN model is provided in [15-18].

Structure of the Legendre neural network $[19,20]$ (LeNN) (Figure 1(c)) is similar to FLANN. In contrast 
TABLE 4: Simulation study of tipper noise.

\begin{tabular}{|c|c|c|c|c|c|c|c|c|}
\hline \multirow{2}{*}{$\begin{array}{l}\text { Distance from the } \\
\text { source (meters) }\end{array}$} & \multirow{2}{*}{$\begin{array}{l}\text { Measured field } \\
\text { data }(\mathrm{dBA})\end{array}$} & \multicolumn{4}{|c|}{ Prediction result (dBA) } & \multicolumn{2}{|c|}{ Average percentage error (dBA) } & \multirow[b]{2}{*}{ LeNN } \\
\hline & & VDI & PPN & FLANN & LeNN & PPN & FLANN & \\
\hline 1 & 100.9000 & 94.2919 & 74.1198 & 85.6989 & 81.4871 & & & \\
\hline 2 & 99.7000 & 93.0828 & 74.1198 & 93.0404 & 81.4871 & & & \\
\hline 3 & 98.6000 & 91.9738 & 74.1198 & 94.5178 & 81.4871 & & & \\
\hline 4 & 97.5000 & 90.8648 & 74.1198 & 92.4313 & 81.4871 & & & \\
\hline 5 & 96.5000 & 89.8559 & 74.1198 & 87.9020 & 81.4871 & & & \\
\hline 6 & 96.2000 & 89.5469 & 85.8609 & 96.5394 & 94.5712 & & & \\
\hline 7 & 95.8000 & 89.1380 & 85.8609 & 93.3190 & 94.5712 & & & \\
\hline 8 & 94.8000 & 88.1291 & 85.8609 & 93.3190 & 94.5712 & & & \\
\hline 9 & 94.3000 & 87.6202 & 85.8609 & 94.0383 & 94.5712 & & & \\
\hline 10 & 93.7000 & 87.0113 & 85.8609 & 91.0641 & 94.5712 & & & \\
\hline 11 & 92.8000 & 86.1025 & 85.8609 & 84.6752 & 94.5712 & & & \\
\hline 12 & 90.6000 & 83.8937 & 85.8609 & 88.3686 & 94.5712 & & & \\
\hline 13 & 89.5000 & 82.8848 & 85.8609 & 88.1936 & 94.5712 & & & \\
\hline 14 & 88.4000 & 81.6760 & 74.1198 & 80.8651 & 81.4871 & & & \\
\hline 15 & 86.8000 & 80.0672 & 64.8344 & 74.8196 & 70.1930 & 13.59 & 4.36 & 10.03 \\
\hline 16 & 86.2000 & 79.4585 & 64.8344 & 74.8196 & 70.1930 & & & \\
\hline 17 & 85.8000 & 79.0497 & 64.8344 & 78.0400 & 70.1930 & & & \\
\hline 18 & 85.2000 & 78.4410 & 64.8344 & 77.8650 & 70.1930 & & & \\
\hline 19 & 85.2000 & 78.4323 & 64.8344 & 77.1458 & 70.1930 & & & \\
\hline 20 & 84.7000 & 77.9236 & 64.8344 & 77.1458 & 70.1930 & & & \\
\hline 21 & 84.5000 & 77.7149 & 64.8344 & 74.5798 & 70.1930 & & & \\
\hline 22 & 83.8000 & 77.0063 & 62.5730 & 73.8606 & 70.1930 & & & \\
\hline 23 & 83.5000 & 76.6976 & 62.5730 & 73.8606 & 70.1930 & & & \\
\hline 24 & 83.5000 & 76.6890 & 61.4715 & 70.6402 & 70.1930 & & & \\
\hline 25 & 83.2000 & 76.3804 & 61.4715 & 72.5517 & 66.3679 & & & \\
\hline 26 & 82.8000 & 75.9718 & 63.5968 & 74.1068 & 66.3679 & & & \\
\hline 27 & 82.6000 & 75.7632 & 59.7479 & 70.8864 & 66.3679 & & & \\
\hline 28 & 82.2000 & 75.3547 & 60.3181 & 71.0614 & 67.0982 & & & \\
\hline 29 & 82.2000 & 75.3461 & 60.3181 & 71.7806 & 67.3327 & & & \\
\hline 30 & 82.2000 & 75.3376 & 72.2342 & 82.3295 & 82.9287 & & & \\
\hline
\end{tabular}

to FLANN, in which trigonometric functions are used in the functional expansion, LeNN uses Legendre orthogonal functions. LeNN offers faster training compared to FLANN. The performance of this model may vary from problem to problem. The Legendre polynomials are denoted by $L_{n}(X)$, where $n$ is the order and $-1<x<1$ is the argument of the polynomial. The zero and the first-order Legendre polynomials are, respectively, given by $L_{0}(x)=1$ and $L_{1}(x)=x$. The higher order polynomials are given by $L_{2}(x)=1 / 2\left(3 x^{2}-1\right), L_{3}(x)=1 / 2\left(5 x^{3}-3 x\right)$ and so forth. Polynomials are generated by using the following mathematical expression:

$$
L_{n+1}(x)=\frac{1}{n+1}\left[(2 n+1) x L_{n}(x)-n L_{n-1}(x)\right] .
$$

Similar to FLANN, the two-dimensional input pattern $X=\left[x_{1} x_{2}\right]^{T}$ is enhanced to a seven dimensional pattern by Legendre functional expansion $X^{e}=\left[1, L_{1}\left(x_{1}\right), L_{2}\left(x_{1}\right)\right.$, $\left.L_{3}\left(x_{1}\right), L_{1}\left(x_{2}\right), L_{2}\left(x_{2}\right), L_{3}\left(x_{2}\right)\right]$. For Legendre neural network, the training is carried out in the same manner as FLANN and PPN. In all models, supervised learning is used. As in normal artificial neural network techniques, the presence of hidden layers increases the complexity in the real-time system, therefore, FLANN and LeNN is suitably used at here due to less computational cost.

\section{Development of Functional Link Artificial Neural Network Based-Noise Prediction Model}

The functional link artificial neural network-based noise prediction models consist of two input parameters: sound power level $\left({ }^{1} x_{k}\right)$ and distance $\left({ }^{2} x_{k}\right)$. The inputs patterns are ${ }^{1} x_{1}(k),{ }^{1} x_{2}(k),{ }^{1} x_{3}(k) \ldots .{ }^{1} x_{n}(k) \in R,{ }^{2} x_{1}(k),{ }^{2} x_{2}(k)$, 


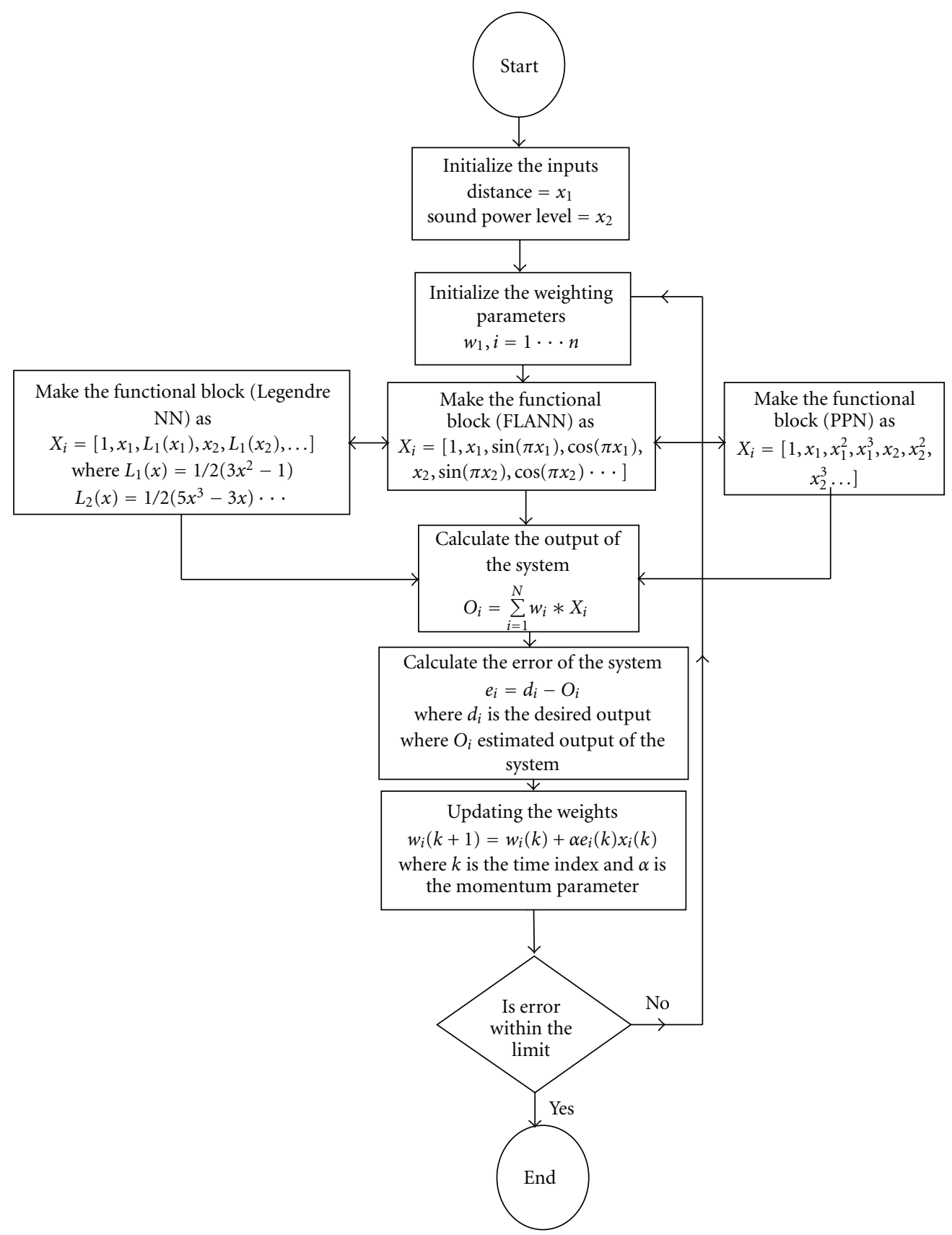

FIGURE 2: Systematic algorithm of functional based neural network based noise prediction models.

${ }^{2} x_{3}(k) \ldots{ }^{2} x_{n}(k)$ and the desired output patterns are: $d_{1}(k)$, $d_{2}(k), d_{3}(k) \cdots d_{n}(k) \in R$. During training period, the desired network output was calculated with VDI-2714 noise prediction model. Since the procedures of these three models were similar; therefore, one algorithm is presented here to emphasize the development of functional-based neural network-based noise prediction models. Figure 2 graphically represent the algorithm of functional-based neural networkbased noise prediction models.

Step 1. Initialize the inputs distance $=x_{1, i},(i=1, \ldots, n)$, sound power level $=x_{2, j}(j=1, \ldots, m)$, where $n$ and $m$ are the number of input pattern and an error tolerance parameter $\varepsilon>0$. The dimension of $m$ and $n$ should be same.

Step 2. Randomly select the initial values of the weight vectors $w_{i}$, for $i=1,2, \ldots l$, where " $i$ " is the number of functional elements.

Step 3 (Initialization). All the weights $w_{i}$ were initialized to random number and given as $w_{i}(0)$

$$
w_{i} \longleftarrow w_{i}(0) .
$$


TABle 5: Simulation study of dozer noise.

\begin{tabular}{|c|c|c|c|c|c|c|c|c|}
\hline \multirow{2}{*}{$\begin{array}{l}\text { Distance from the } \\
\text { source (meters) }\end{array}$} & \multirow{2}{*}{$\begin{array}{l}\text { Measured field } \\
\text { data (dBA) }\end{array}$} & \multicolumn{4}{|c|}{ Prediction result (dBA) } & \multicolumn{2}{|c|}{ Average percentage error (dBA) } & \multirow[b]{2}{*}{ LeNN } \\
\hline & & VDI & PPN & FLANN & LeNN & PPN & FLANN & \\
\hline 1 & 100.5000 & 93.8919 & 74.1198 & 85.6989 & 81.4871 & & & \\
\hline 2 & 100.2000 & 93.5828 & 74.1198 & 93.0404 & 81.4871 & & & \\
\hline 3 & 98.2000 & 91.5738 & 74.1198 & 94.5178 & 81.4871 & & & \\
\hline 4 & 97.5000 & 90.8648 & 74.1198 & 92.4313 & 81.4871 & & & \\
\hline 5 & 96.7000 & 90.0559 & 74.1198 & 87.9020 & 81.4871 & & & \\
\hline 6 & 95.4000 & 88.7469 & 74.1198 & 85.9905 & 81.4871 & & & \\
\hline 7 & 94.8000 & 88.1380 & 85.8609 & 93.3190 & 94.5712 & & & \\
\hline 8 & 94.2000 & 87.5291 & 85.8609 & 93.3190 & 94.5712 & & & \\
\hline 9 & 93.6000 & 86.9202 & 85.8609 & 94.0383 & 94.5712 & & & \\
\hline 10 & 92.5000 & 85.8113 & 85.8609 & 94.0383 & 94.5712 & & & \\
\hline 11 & 91.8000 & 85.1025 & 85.8609 & 87.6493 & 94.5712 & & & \\
\hline 12 & 89.6000 & 82.8937 & 74.1198 & 77.8197 & 81.4871 & & & \\
\hline 13 & 89.3000 & 82.5848 & 85.8609 & 88.1936 & 94.5712 & & & \\
\hline 14 & 88.8000 & 82.0760 & 85.8609 & 91.4140 & 94.5712 & & & \\
\hline 15 & 88.2000 & 81.4672 & 76.5756 & 85.3685 & 85.3813 & 10.94 & 6.32 & 9.53 \\
\hline 16 & 87.9000 & 81.1585 & 76.5756 & 85.3685 & 85.3813 & & & \\
\hline 17 & 87.4000 & 80.6497 & 76.5756 & 88.5889 & 85.3813 & & & \\
\hline 18 & 86.6000 & 79.8410 & 76.5756 & 88.4139 & 85.3813 & & & \\
\hline 19 & 85.5000 & 78.7323 & 64.8344 & 77.1458 & 70.1930 & & & \\
\hline 20 & 85.5000 & 78.7236 & 76.5756 & 87.6947 & 85.3813 & & & \\
\hline 21 & 84.8000 & 78.0149 & 64.8344 & 74.5798 & 70.1930 & & & \\
\hline 22 & 84.3000 & 77.5063 & 62.5730 & 73.8606 & 70.1930 & & & \\
\hline 23 & 84.2000 & 77.3976 & 62.5730 & 73.8606 & 70.1930 & & & \\
\hline 24 & 83.8000 & 76.9890 & 61.4715 & 70.6402 & 70.1930 & & & \\
\hline 25 & 83.5000 & 76.6804 & 61.4715 & 72.5517 & 66.3679 & & & \\
\hline 26 & 83.5000 & 76.6718 & 75.3380 & 84.6557 & 82.0894 & & & \\
\hline 27 & 82.8000 & 75.9632 & 59.7479 & 70.8864 & 66.3679 & & & \\
\hline 28 & 82.5000 & 75.6547 & 60.3181 & 71.0614 & 67.0982 & & & \\
\hline 29 & 82.4000 & 75.5461 & 72.0592 & 82.3295 & 82.9287 & & & \\
\hline 30 & 82.4000 & 75.5376 & 72.2342 & 82.3295 & 82.9287 & & & \\
\hline
\end{tabular}

Step 4 (Produce functional blocks). For FLANN the functional block is made as follows:

$$
X_{i}=\left[1, x_{1}, \sin \left(\pi x_{1}\right), \cos \left(\pi x_{1}\right), x_{2}, \sin \left(\pi x_{2}\right), \cos \left(\pi x_{2}\right) \ldots\right],
$$

For PPN the functional block is made as follows:

$$
X_{i}=\left[1, x_{1}, x_{1}^{2}, x_{1}^{3}, x_{2}, x_{2}^{2}, x_{2}^{3} \ldots\right]
$$

for LeNN the functional block is made as follows:

$$
X_{i}=\left[1, x_{1}, L_{1}\left(x_{1}\right), x_{2}, L_{1}\left(x_{2}\right), \ldots\right],
$$

where $L_{1}(x)=(1 / 2)\left(3 x^{2}-1\right), L_{2}(x)=(1 / 2)\left(5 x^{3}-3 x\right)$, and so forth.
Step 5 (Calculation of the system outputs). For functional based neural network models, the output was calculated as follows:

$$
O_{i}=\tanh \left(\sum_{i=1}^{N} w_{i} \times X_{i}\right) .
$$

Step 6 (Calculation of the output error). The error was calculated as $e_{i}=d_{i}-O_{i}$. It may be seen that the network produces a scalar output.

Step 7 (Updating the weight vectors). The weight matrixes are updated next using the following relationship:

$$
w_{i}(k+1)=w_{i}(k)+\alpha e_{i}(k) X_{i}(k),
$$

where $k$ is the time index and $\alpha$ is the momentum parameter.

Step 8. If error $\leq \varepsilon(0.01)$ then go to Step 8 otherwise, go to Step 3. 


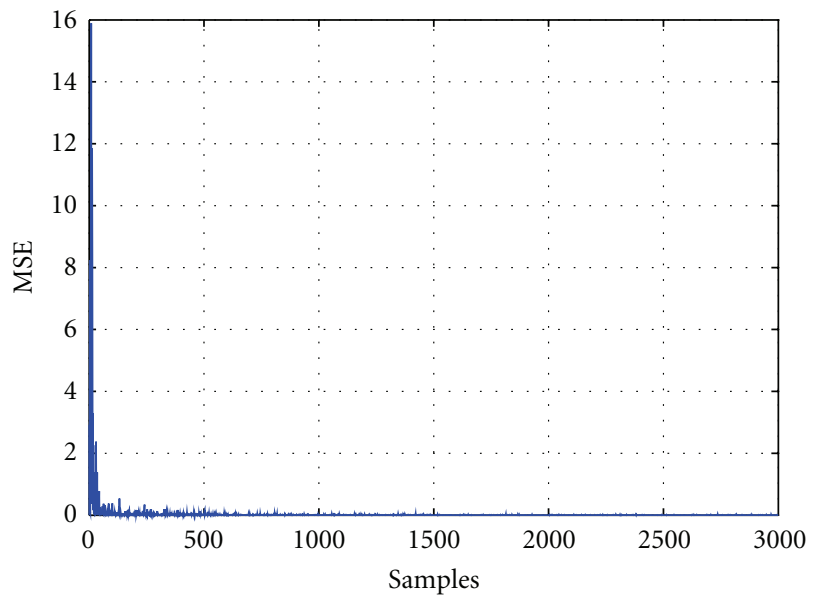

FIGURE 3: Mean square plot of FLANN based noise prediction model for 3000 iteration.

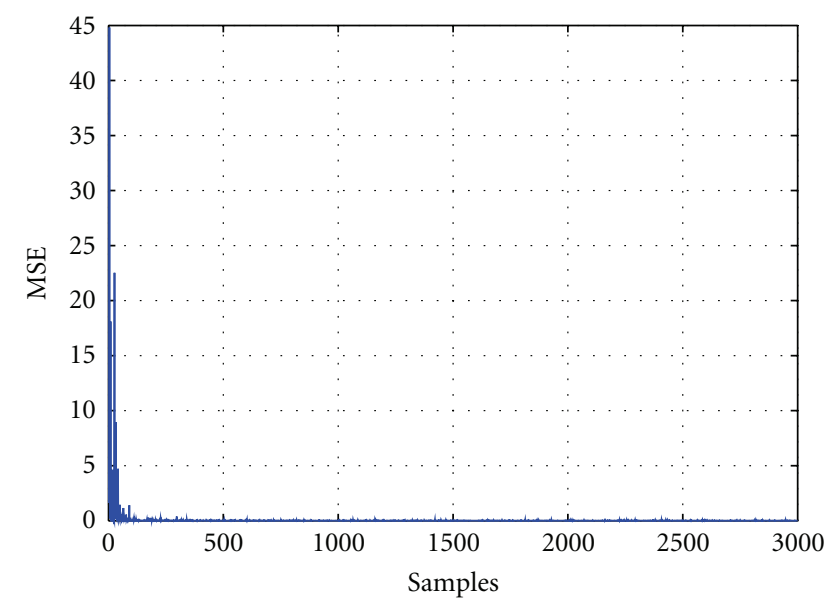

FIGURE 4: Mean square plot of PPN based noise prediction model for 3000 iteration.

Step 9. After the, learning is complete, the weights were fixed, and the network can be used for testing.

\section{Simulation Result and Discussion}

The proposed system models for noise prediction were validated using simulation studies. The studies were carried out by using MATLAB simulation environment. For validation of the models, the noise data was collected from Balaram opencast coal mine of Mahanadi Coalfields Limited (MCL), Talcher (Orissa, India). The test data was measured using Brüel and Kjaer 2239 (Denmark) precision sound level meter. From the measured parameter, VDI-2714 gives prediction by calculating all the sound attenuations in " $\mathrm{dB}(\mathrm{A})$ " not in octave frequency band. SPL of the different machineries from the above mine was collected. These machineries include Shovel $\left(10 \mathrm{~m}^{3}\right.$ bucket capacity), dozer $(410 \mathrm{HP})$, tipper (10 T-160 HP), grader $(220 \mathrm{HP})$, and dumper $(85 \mathrm{~T})$.

According to Figure 2, the stepwise procedures were required to design the model. In this problem, the system

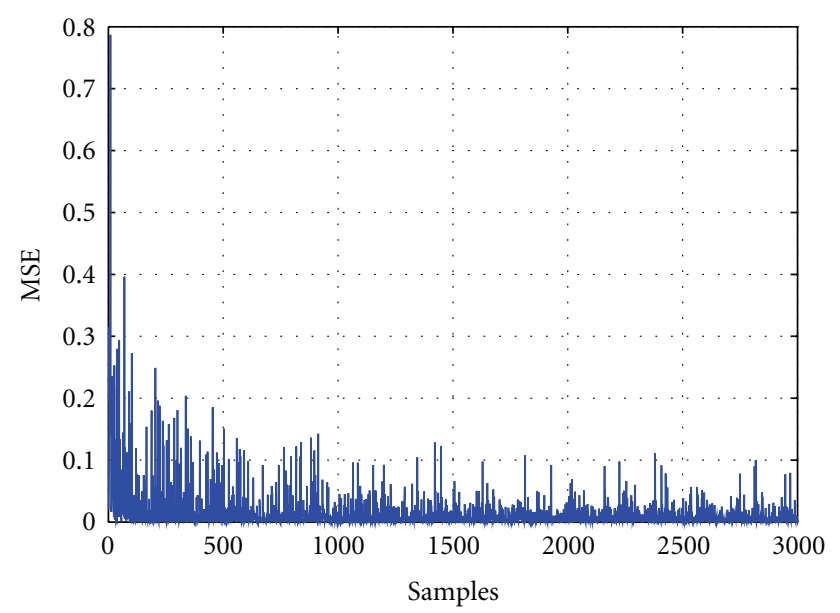

FIGURE 5: Mean square plot of LeNN based noise prediction model for 3000 iteration.

is a MISO (multi input and output system) system. The system architectures of these proposed functional link-based noise prediction models are the same, whereas only the input pattern or functional blocks are different. To design these models, total number of 3200 dataset were selected. Out of 3200,3000 dataset were selected for training process and 200 data were selected for testing process. In this proposed systems, iteration based training methods were applied. The mean square error (MSE) plot of FLANN-based noise prediction model is represented in Figure 3, where Figures 4 and 5 are represented PPN and LeNN noise prediction models. Performance of these models for 200 testing samples or validation samples was represented from Figures 6, 7, and 8.The average percentage error (APE) was used as the performance index and was calculated as

\section{Average Percentage Error}

$$
=\frac{1}{N} \sum_{i=1}^{N} \frac{\left(\mathrm{VDI}_{i}-\text { Estimated }_{i}\right)^{2}}{\mathrm{VDI}_{i}} \times 100 .
$$

Tables 1, 2, 3, 4, and 5 summarizes the results for noise prediction by proposed models and compares it with standard VDI-2714 noise prediction model for all selected opencast machineries. From these tables it can be seen that the proposed PPN, FLANN and LeNN models provided average percentage error of $7.03,5.68$, and 8.42 , respectively, for shovel. For dumper, the average percentage errors were 7.27, 5.77, and 8.20; for Grader, the APE were 9.56, 6.15, and 9.76; for Tipper, APE for three systems were 13.59, 4.36 , and 10.03 , respectively. The average percentage errors of the dozer were found as $10.94,6.32$, and 9.53 respectively. From the simulation studies, it was observed that the average percentage error of FLANN model was lower than the other two models.

\section{Conclusion}

This paper introduced the idea of designing noise prediction model for opencast mining machineries using functional link 


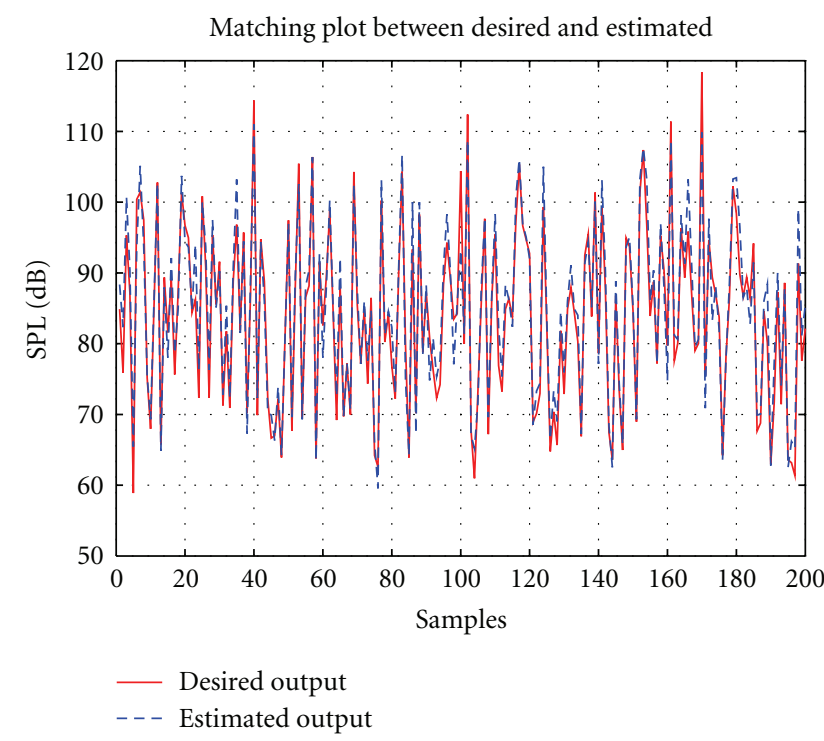

Figure 6: Prediction performance of FLANN system for 200 samples.

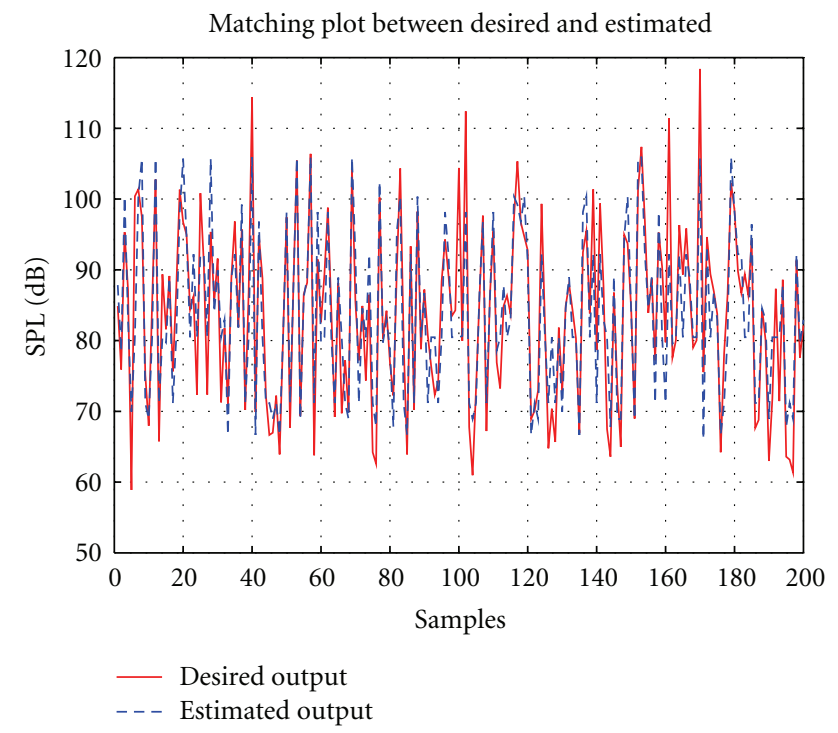

FIgURE 7: Prediction performance of PPN system for 200 samples.

artificial neural network systems. From the present study, it was observed that the average percentage error using FLANN lower than PPN and LeNN systems for all the machineries. These functional link artificial neural network based noise prediction models can be useful tools for mining engineers to estimate the actual noise condition of the machineries accurately.

\section{Acknowledgments}

The authors like to thank the anonymous referees for their valuable comments and words of encouragement. Their comments helped to improve the clarity of this paper.

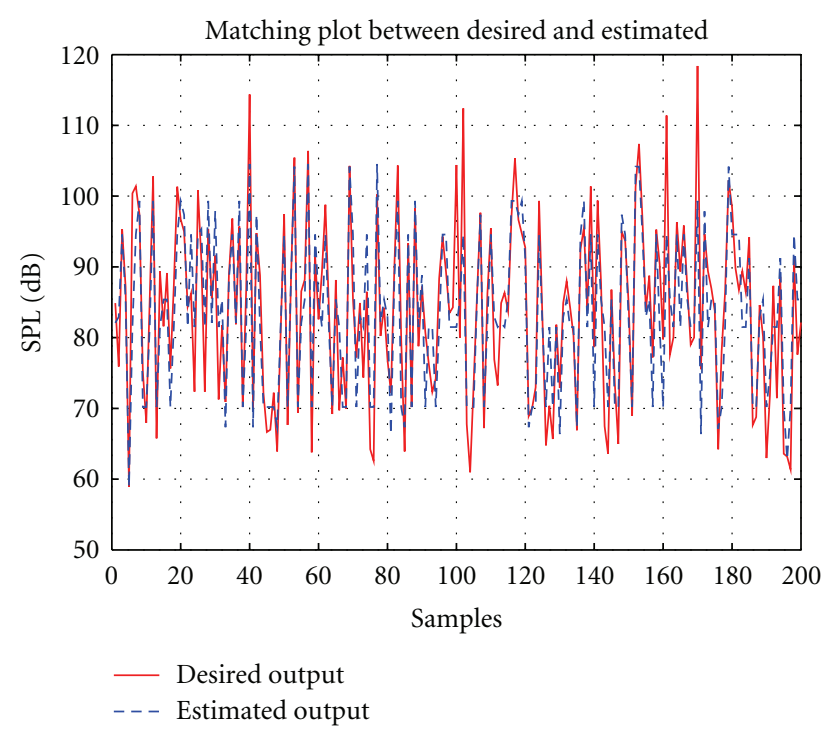

Figure 8: Prediction performance of LeNN system for 200 samples.

\section{References}

[1] C. Sensogut, "Occupational noise in mines and its control-a case study," Polish Journal of Environmental Studies, vol. 16, no. 6, pp. 939-942, 2007.

[2] K. J. Marsh, "The CONCAWE model for calculating the propagation of noise from open-air industrial plants," Applied Acoustics, vol. 15, no. 6, pp. 411-428, 1982.

[3] K. Pathak, S. Durucan, and S. Kunimatsu, "Activity accounting technique for environmental noise assessment in mechanized surface mining," Environmental Monitoring and Assessment, vol. 56, no. 3, pp. 241-256, 1999.

[4] N. K. Mohalik and A. K. Pal, "Development of a noise model with respect to sound propagation and its application to a mining complex," Noise and Vibration Worldwide, vol. 33, no. 2, pp. 8-16, 2003.

[5] R. Tonin, "Estimating noise level from petrochemical plants, mines and industrial complexes," Acoustics Australia, vol. 13, pp. 59-67, 1985.

[6] A. K. Pal, Y. Mishra, S. Mitra, and N. C. Saxena, "Noise model for mining complex-a case discussion," in Proceedings of the 2nd World Mining Environment Congress, pp. 1-10, Katowice, Poland, 1997.

[7] R. E. Rabeiy, M. Mohamed, and W. Gomma, "Study on the noise prediction in mining and industrial plants," Assiut University Bulletin for Environmental Researches, vol. 7, no. 2, pp. 77-89, 2004.

[8] K. Pathak, S. Durucan, and A. Korre, "Development of an air attenuation model for noise prediction in surface mines and quarries," International Journal of Surface Mining, Reclamation and Environment, vol. 14, no. 3, pp. 223-234, 2000.

[9] J.-S. R. Jang, C.-T. Sun, and E. Mizutan, Neuro-Fuzzy and Soft Computing, Prentice Hall of India Private Limited, New Delhi, India, 2005.

[10] S. Haykin, Adaptive Filter Theory, Pearson Education, Inc., New Delhi, India, 2002.

[11] S. Haykin, Neural Networks: A Comprehensive Foundation, Prentice-Hall, Reading, Mass, USA, 1994.

[12] M. Hagan, H. Demuth, and M. Beale, Neural Network Design, Thomson Learning, New Delhi, India, 2003. 
[13] K. S. Narendra and K. Parthasarathy, "Identification and control of dynamical systems using neural networks," IEEE Transactions on Neural Networks, vol. 1, no. 1, pp. 4-27, 1990.

[14] Z. Xiang, G. Bi, and T. Le-Ngoc, "Polynomial perceptrons and their applications to fading channel equalization and cochannel interference suppression," IEEE Transactions on Signal Processing, vol. 42, no. 9, pp. 2470-2479, 1994.

[15] J. C. Patra and R. N. Pal, "Functional link artificial neural network-based adaptive channel equalization of nonlinear channels with QAM signal," in Proceedings of the IEEE International Conference on Systems, Man and Cybernetics, vol. 3, pp. 2081-2086, 1995.

[16] J. C. Patra and R. N. Pal, "A functional link artificial neural network for adaptive channel equalization," Signal Processing, vol. 43, no. 2, pp. 181-195, 1995.

[17] J. C. Patra, R. N. Pal, R. Baliarsingh, and G. Panda, "Nonlinear channel equalization for QAM signal constellation using artificial neural networks," IEEE Transactions on Systems, Man, and Cybernetics, Part B, vol. 29, no. 2, pp. 262-271, 1999.

[18] C. H. Chen, C. J. Lin, and C. T. Lin, "A functional-linkbased neurofuzzy network for nonlinear system control," IEEE Transactions on Fuzzy Systems, vol. 16, no. 5, pp. 1362-1378, 2008.

[19] J. C. Patra, W. C. Chin, P. K. Meher, and G. Chakraborty, "Legendre-FLANN-based nonlinear channel equalization in wireless communication system," in Proceedings of the IEEE International Conference on Systems, Man and Cybernetics, pp. 1826-1831, Singapore, October 2008.

[20] J. C. Patra, P. K. Meher, and G. Chakraborty, "Nonlinear channel equalization for wireless communication systems using Legendre neural networks," Signal Processing, vol. 89, no. 11, pp. 2251-2262, 2009. 

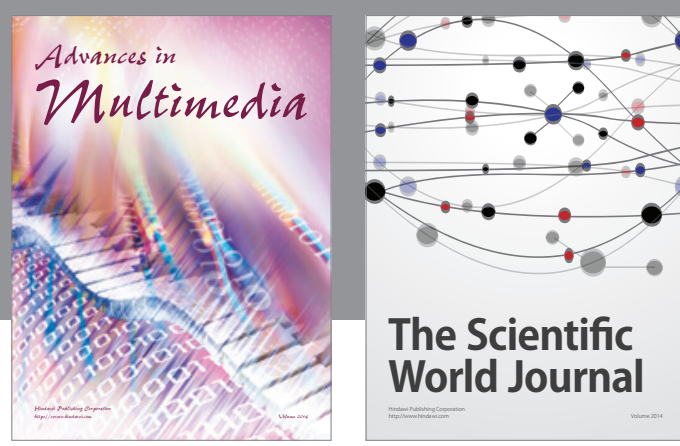

The Scientific World Journal
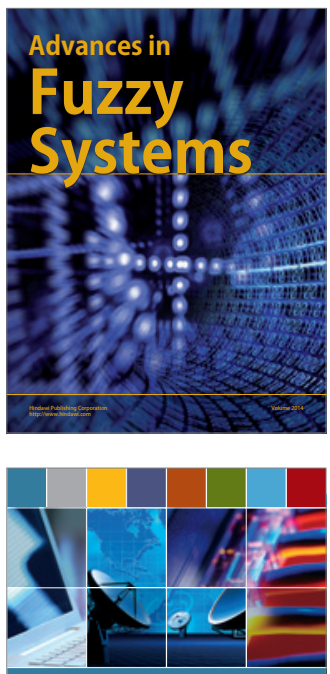

Computer Networks and Communications
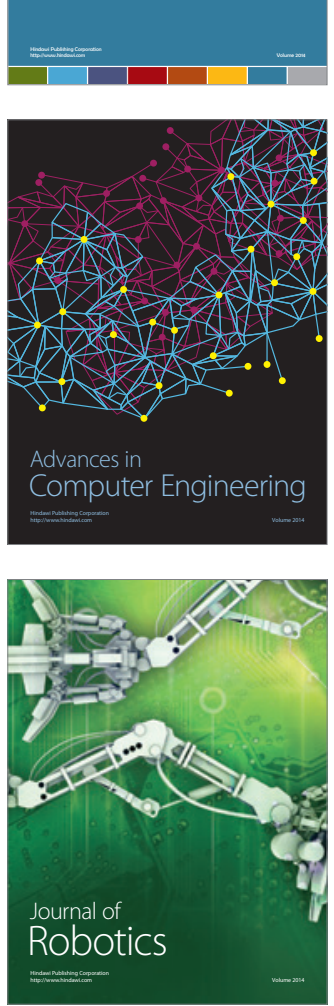
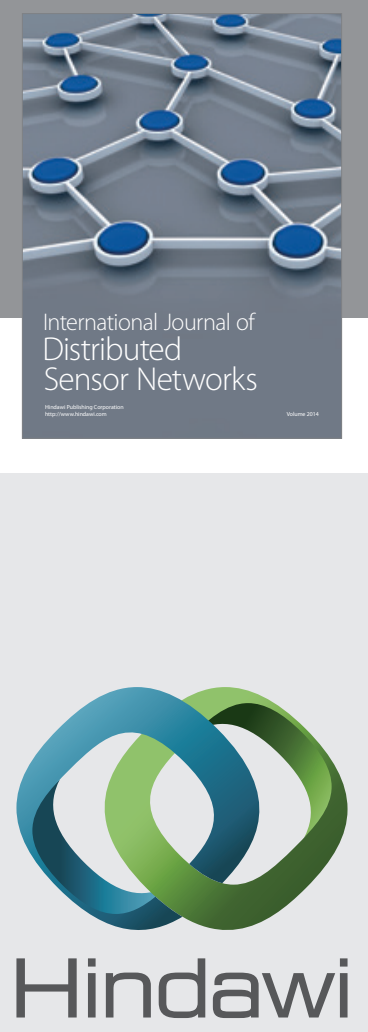

Submit your manuscripts at

http://www.hindawi.com
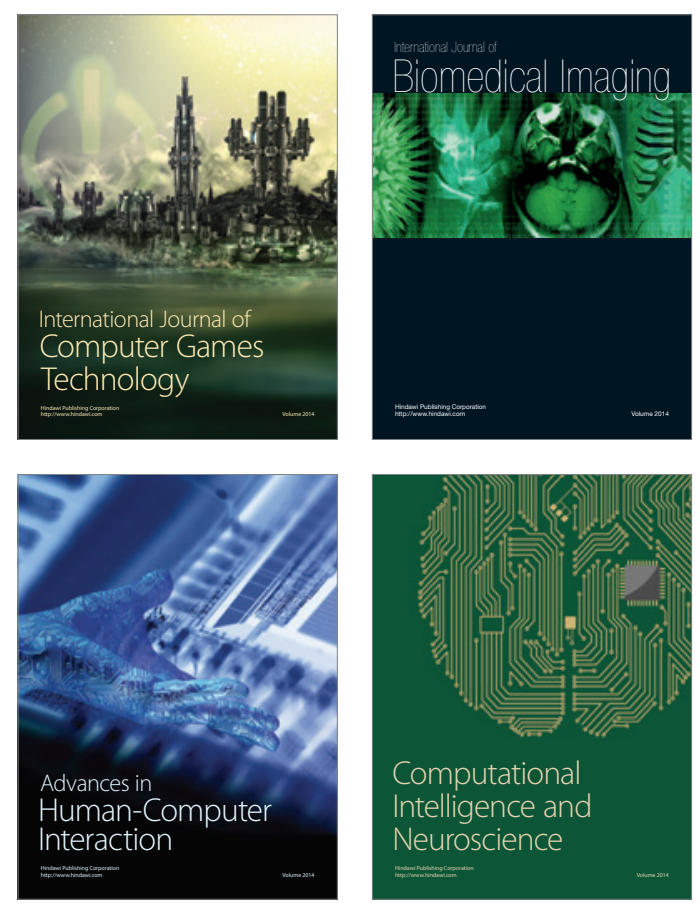
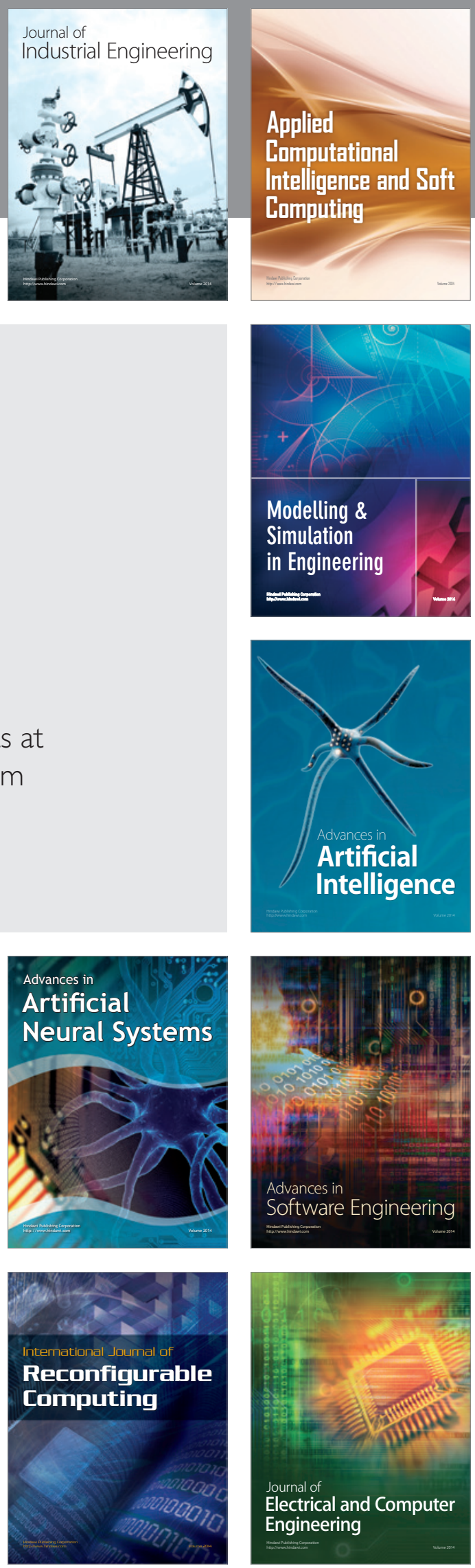